\title{
Preliminary study on ultrasound-guided prostate biopsy specimen scores
}

\author{
CHENG LI, WEIWEI ZHAN, MINGUANG ZHANG, FANGXIU LUO, YAN WANG and BIN ZHENG \\ Department of Ultrasound, Ruijin Hospital North Affiliated to Shanghai Jiao Tong University School of Medicine, \\ Shanghai 200025, P.R. China
}

Received February 22, 2016; Accepted February 24, 2017

DOI: $10.3892 /$ etm.2017.4800

\begin{abstract}
Prostate cancer (PCa) is the second most frequently diagnosed cancer in males worldwide and resulted in $\sim 258,000$ cases of cancer-associated mortality in 2008 . The present study visually determined the pathology scores of PCa specimens by taking into account five characteristics, including the hardness, color, plumpness, transparency and uniformity of specimens. The current study also aimed to identify the association between pathology scores and prostate specific antigen (PSA) levels, in order to reduce the complications caused by punctures and elevate the specimen positive rates. A total of 1,608 specimens from 268 patients were analyzed by one sonographer, one urologist and one pathologist. A standard pathological examination was performed on the PCa biopsy specimens and specimen scores were recorded under double-blinded conditions. A receiver operator characteristic curve identified a linear correlation between the visually determined score and PSA levels $(\mathrm{r}=0.255 ; \mathrm{P}<0.001)$. Furthermore, logistic regression analysis indicated that the visually determined score and PSA were correlated with the diagnosis of PCa. Additionally, the authenticity of the visually determined score was higher than PSA in the diagnosis of PCa, with the best sensitivity and specificity of the visually determined scores used to predict PCa being 0.817 and 0.931 , respectively.
\end{abstract}

\section{Introduction}

Prostate cancer ( $\mathrm{PCa}$ ) is the second most frequent type of cancer diagnosed in males worldwide. The cancer can invade bones and lymph nodes and it is the second leading cause of cancer-related mortality in men (1-4). There are no obvious

Correspondence to: Dr Weiwei Zhan, Department of Ultrasound, Ruijin Hospital North Affiliated to Shanghai Jiao Tong University School of Medicine, 197 Ruijin No. 2 Road, Shanghai 200025, P.R. China

E-mail: zhanweiweish@sina.com

Key words: prostate biopsy, visually determined score, prostate specific antigen symptoms when PCa first occurs, however in the later stages the patient will experience difficulty urinating, blood in the urine, or pain in the pelvis or back when urinating. There were 910,000 new cases of PCa and 258,000 cases of PCa-associated mortality in 2008 and it is estimated that the incidence of PCa may double by 2030 (5). Prostate specific antigen (PSA; clone ER-PR8, cytoplasmic staining) was initially identified $\sim 30$ years ago and is currently the most useful biomarker for cancer detection. Furthermore, serum PSA levels are widely applied to distinguish between $\mathrm{PCa}$ and benign prostatic hyperplasia $(6,7)$ Additionally, in patients with $\mathrm{PCa}$, it is crucial to accurately assess tumor aggressiveness to guide treatment decisions, as well as determine patient prognosis. Transrectal ultrasound (TRUS)-guided biopsy, typically performed with blind finger-guided intervention under general anesthesia to a directed lesion, is the standard procedure used for $\mathrm{PCa}$ diagnosis (8).

Diverse sampling devices and biopsy techniques, including X-ray examination, ultrasonic testing, computed tomography and magnetic resonance imaging, have been utilized to reach an optimum performance-hazard ratio. The dilemma between higher sensitivity and higher morbidity has been ongoing since the first description of the sextant biopsy scheme, which is an accurate and basic method for diagnosing PCa using a fine needle inserted into the prostate, by Hodge et al (9). Techniques that are more sensitive increase the likelihood of morbidity in patients (10). Although the sextant biopsy scheme is applied in multiple centers, it has been recommended that a minimum of 10-12 core biopsies should be taken and this method is currently adopted by the majority of urologists (11). The optimal number of core biopsies remains unknown, however, it has been confirmed that increasing the number of cores notably improves cancer detection. The use of 12-21 cores markedly increases cancer detection rates compared with sextant biopsies $(12,13)$. However, this also increases the incidence of complications, and may even cause some serious complications (14).

The aim of the current study was to achieve the highest cancer detection rate using the minimal number of cores. Therefore, pathology standard examination scoring was used to analyze five different aspects of the specimens, including hardness, color, plumpness, transparency and uniformity. Additionally, the association between pathology scores and PSA levels was investigated. 


\section{Patients and methods}

Patients. A total of 268 patients aged $71 \pm 8$ years old and suspected of having PCa were admitted and treated at Ruijin Hospital (Shanghai, China) between January 2013 and December 2013. Indications for prostate biopsy were as follows: Serum PSA $>4 \mathrm{ng} / \mathrm{ml}$, or ultrasound or MRI found suspicious nodules, or clinical anus examination identified hard nodules. All patients were hospitalized, underwent routine hospitalization tests and coagulation index detection. A total of 6 prostate biopsy specimens were obtained from each patient and all specimens possessed pathological results. Prior written and informed consent was obtained from each participant and the present study was approved by the Ethics Committee of Ruijin Hospital Affiliated to Shanghai Jiaotong University, School of Medicine (Shanghai, China).

Serum PSA evaluation. Patients should have not ejaculated within $24 \mathrm{~h}$, conducted bladder microscopy or catheterization within $48 \mathrm{~h}$, accepted prostate massage within 1 week or performed prostate puncture within 1 month prior to serum PSA evaluation. This was to limit the presence of confounding factors that may have affected PSA levels. Following overnight fasting, patients had $3 \mathrm{ml}$ forearm venous blood drawn in the morning and were inspected using a PSA ELISA kit (cat. no. KA0208; Abnova, Taipei, Taiwan R.O.C.) and analyzed using an automated immunochemistry analyzer (Abbott Laboratories, Abbott Park, IL, USA).

Puncture method. A MyLab ${ }^{\mathrm{TM}} 60$ diasonograph (Esaote, Genova, Italy), rectal biplanar probe (Esaote), automated biopsy gun (Bard Biopsy Systems, Tempe, AZ, USA) and 16-gauge (G) Tru-cut biopsy needle (Bard Biopsy Systems) were used in the present study. The length of the specimen slot was set to $2.2 \mathrm{~cm}$. Patients were asked to lift up the scrotum to fully expose the perineum, then transverse scanning was performed using a convex array probe to measure the prostate anteroposterior and transverse diameters. Axial scanning was performed by linear array probe to measure prostate axial diameter. This allowed for the detection of nodules, cysts or calcification, and the selection of an appropriate puncture site.

Following perineum disinfection, infiltration anesthesia was administered with $5 \mathrm{ml} 1 \%$ lidocaine (Century Pharmaceutical Co., Ltd., Tianjin, China) on each side of the midcourt line, which was at the middle of the urethral bulb and ischial tuberosity, and 1.5-2.0 cm away from the anus. The sextant biopsy scheme was subsequently utilized to obtain prostate biopsy specimens. Puncture points were on the tip of the prostate near the midline sagittal section, as well as the middle and bottom of the prostrate, for a total of six puncture points per patient.

Visual scoring method. One sonographer, one urologist and one physician pathologist evaluated all the specimens. Specimens were scored based on five aspects: Hardness, color, plumpness, transparency and uniformity, and were given a score of either 0 or 1 (Table I). Specimen scores were recorded under double-blinded conditions and thereafter, the averages of six specimen ratings were calculated.
Hematoxylin and eosin $(H \& E)$ staining. Prostate tissues were fixed with $4 \%$ paraformaldehyde at room temperature for $24 \mathrm{~h}$, embedded in paraffin and then sliced into sections $(6 \mu \mathrm{m})$. Briefly, slices were dewaxed with xylene and rehydrated using graded alcohols. Following rinsing with tap water and distilled water, slices were stained with hematoxylin for 3-5 min. Slices were washed with tap water and stained with eosin for 1-4 min and subsequently underwent dehydration and differentiation with $\mathrm{HCl}$ in $70 \%$ alcohol. Slices were then studied using a light microscope.

Statistical analysis. Quantitative data were expressed as the mean \pm standard error of the mean. Statistical comparisons between the PCa and non-PCa groups were performed using a Student's t-test. Logistic regression and receiver operator characteristic curve (ROC) analysis were used to analyze the sensitivity and specificity of the visually determined score and PSA level. Finally, Spearman correlation was applied to determine the correlation between the visually determined scores and PSA levels. $\mathrm{P}<0.05$ was considered to indicate a statistically significant difference.

\section{Results}

Demographic and clinical characteristics of patients. The average age of the 268 patients with suspected PCa patients was $71 \pm 8$ years old. The indications for prostate biopsy were serum PSA $>4 \mathrm{ng} / \mathrm{ml}$, a suspicious nodule identified by heterogeneous echo that was confirmed by ultrasound/magnetic resonance imaging examination or hard nodules revealed following clinical rectal examination. The demographic and clinical characteristics of all patients are presented in Table II.

$H \& E$ staining. The corresponding $\mathrm{H} \& \mathrm{E}$ staining of the $\mathrm{PCa}$ puncture specimen is presented in Fig. 1. The following features were observed: Irregular glands, glandular fibrous hyperplasia, tumor invasive growth, basal cell disappearance, tumor cell nucleolus appearance, mostly single cell morphology.

Extirpated prostate tumor. A representative extirpated tumor from a PCa patient is presented in Fig. 2. The tumor can be seen with the naked eye. The tumor had many ashen hard nodules from $0.3-1.3 \mathrm{~cm}$ in diameter with some red patches spreading out.

PSA and visually determined score. PSA levels in the PCa group $(99.35 \pm 1.40)$ were significantly higher than those of the control group $(17.12 \pm 0.10$; Fig. $3 \mathrm{~A} ; \mathrm{P}<0.01)$. However, the visually determined score $(3.44 \pm 0.01)$ of the PCa group was also significantly increased compared with the control group $(0.99 \pm 0.01$; Fig. 3B; P $<0.01)$. Correlation analysis was carried out on the biopsy specimen visual grading score and PSA. The correlation coefficient was $0.255(\mathrm{P}<0.001)$, which indicated a linear relationship between the two characteristics.

Logistic regression analysis. Logistic regression analysis verified that upregulated PSA and visually determined scores were two independent determining factors in the diagnosis of PCa (Table III). From the data it was identified that PAS and prostate biopsy specimen visual grading have 
Table I. Specimen score principles.

Specimen score

Aspects

1

0

Hardness Hard

Color

Plumpness

Transparency

Uniformity

\section{White}

Plump and mellow

Pattern of the white mat behind specimens could be recognized clearly

Undoped with other tissues
Soft, naturally bends, naturally deformed by gently pressing with fingers

Other colors

Slender or filamentous

Not transparent

Impure
Table II. Demographic and clinical characteristics of patients.

\begin{tabular}{lc}
\hline Characteristics & Number $(\%)$ \\
\hline Age, years & $71 \pm 8$ \\
PSA & \\
$<4 \mathrm{ng} / \mathrm{ml}$ & 0 \\
Hard nodules examined by ultrasound & $5(1.9)$ \\
Hard nodules examined by MRI & $8(3.0)$ \\
Hard nodules revealed by rectal examination & $8(3.0)$ \\
4-10 ng/ml & $61(22.8)$ \\
10-20 ng/ml & $89(33.2)$ \\
$>20 \mathrm{ng} / \mathrm{ml}$ & $97(36.2)$ \\
Pathology result of puncture & \\
Prostate cancer & $93(34.7)$ \\
Prostatic hyperplasia & $109(40.7)$ \\
PIN & $21(7.8)$ \\
Atypical hyperplasia & $45(16.8)$ \\
\hline
\end{tabular}

PSA, prostate specific antigen; MRI, magnetic resonance imaging; PIN, prostatic intraepithelial neoplasm.

statistical significance to distinguish between prostate cancer and non-prostate cancer patients. Higher PSA (OR=1.045, 95\% $\mathrm{CI}=1.021-1.069, \mathrm{P}<0.001)$ or higher visual grading score $(\mathrm{OR}=14.096,95 \% \mathrm{CI}=6.326-31.409, \mathrm{P}<0.001)$ was correlated with a higher risk of $\mathrm{PCa}$.

ROC curve of PSA and visually determined score. An ROC curve analysis was performed to further investigate the clinical value of PSA and visually determined score in the diagnosis of PCa. As shown in Fig. 4 and Table IV, the area under ROC curve of visual grading biopsy specimens was $0.929(\mathrm{P}<0.001)$. The area under ROC curve of PSA was $0.817(\mathrm{P}<0.001)$. The area under ROC curve for visual grading was significantly higher compared with PSA $(\mathrm{P}<0.05)$. Overall, this indicates that the visually determined score is more accurate than PSA in the clinical diagnosis of $\mathrm{PCa}$.

Sensitivity and specificity of the visually determined score. In the current study, if visually determined score was greater

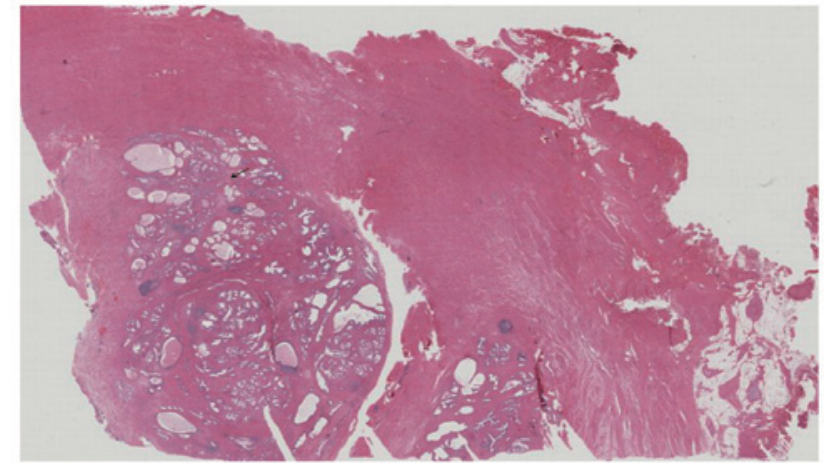

Figure 1. H\&E staining of a prostate cancer specimen. The tumor was taken from a representative PCa patient in the current study and stained with H\&E. The glands have become irregular, and glandular fibrous hyperplasia, tumor invasive growth, basal cell disappearance and tumor cell nucleolus appearance are visible. The tumor cell morphology is mostly single. The arrow indicates acinar adenocarcinoma area of $\mathrm{PCa}$. $\mathrm{H} \& \mathrm{E}$, hematoxylin and eosin.

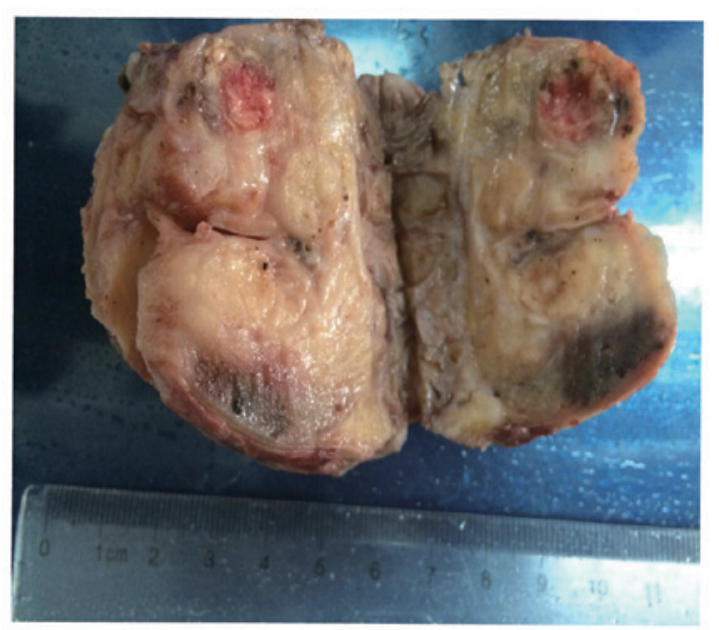

Figure 2. Extirpated prostate tumor. The prostate cancer specimen was taken from a representative PCa patient and has typical characteristics of $\mathrm{PCa}$. The tumor had many ashen hard nodules from $0.3-1.3 \mathrm{~cm}$ in diameter with some red patches spreading out. The specimen size is $8.3 \times 7.2 \times 5 \mathrm{~cm}$.

than 1, 2 or 3 scores, it was considered to be positive. The sensitivity and specificity of the visually determined score in the diagnosis of PCa is presented in Table V. Taking sensitivity and specificity into consideration, diagnosis of PCa using the 
Table III. Logistic regression analysis.

95.0\% CI for $\operatorname{Exp}(\mathrm{B})$

\begin{tabular}{|c|c|c|c|c|c|c|c|}
\hline Indicators & B & S.E. & Wald & P-value & $\operatorname{Exp}(B)$ & Lower & Upper \\
\hline PSA & 0.044 & 0.012 & 13.592 & $<0.001$ & 1.045 & 1.021 & 1.069 \\
\hline Visually determined score & 2.646 & 0.409 & 41.894 & $<0.001$ & 14.096 & 6.326 & 31.409 \\
\hline
\end{tabular}

PSA, prostate specific antigen; S.E., standard error; CI, confidence interval; Exp (B), B coefficient index.

Table IV. Area under the ROC curve of visually determined score and PSA.

$95 \%$ CI of area

\begin{tabular}{lccccc} 
Test result variable(s) & AUC & S.E. & P-value & Lower bound & Upper bound \\
\hline PSA & 0.817 & 0.028 & $<0.001$ & 0.762 & 0.873 \\
Visually determined score & 0.929 & 0.019 & $<0.001$ & 0.892 & 0.967
\end{tabular}

PSA, prostate specific antigen; ROC, receiver operator characteristic curve; AUC, area under curve; S.E.; CI, confidence interval.
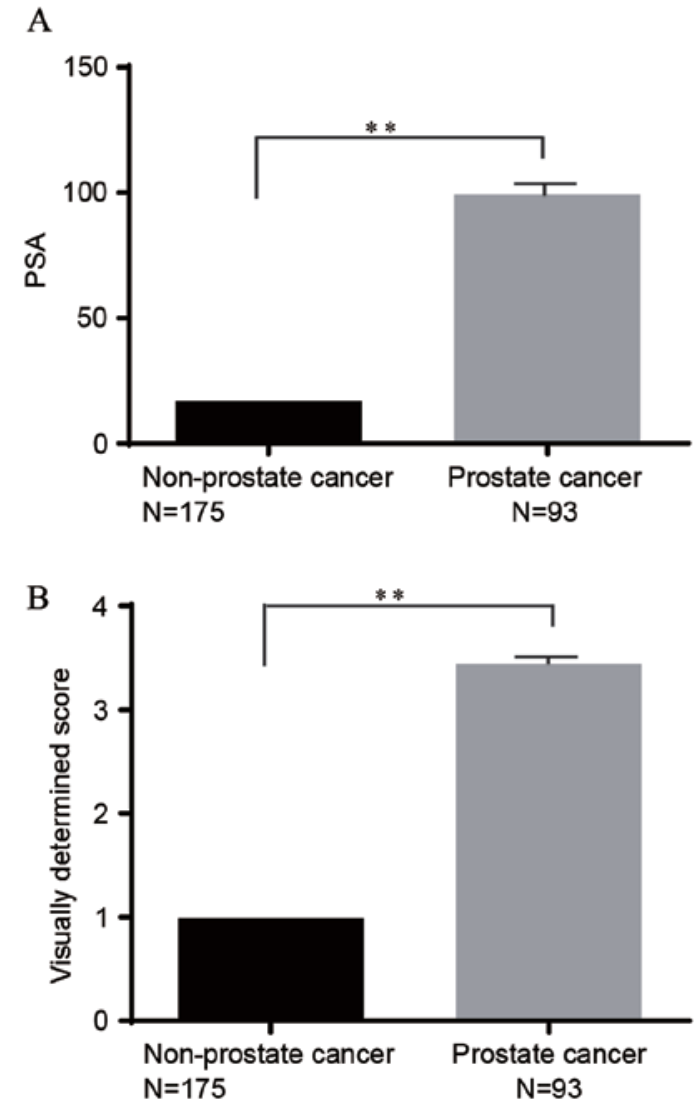

Figure 3. (A) PSA and (B) visually determined score in non-prostate cancer and prostate cancer groups. PSA, prostate specific antigen. ${ }^{* *} \mathrm{P}<0.01$ as indicated.

visual method should take into account 2 scores (the sensitivity is 0.817 and the specificity is 0.931 ), as this has the highest sensitivity and specificity compared with other numbers of scores.
Table V. Sensitivity and specificity of the visually determined score in the diagnosis of prostate cancer.

\begin{tabular}{lcc}
$\begin{array}{l}\text { Diagnostic point of } \\
\text { visually determined score }\end{array}$ & Sensitivity & Specificity \\
\hline 1 & 0.957 & 0.686 \\
2 & 0.817 & 0.931 \\
3 & 0.548 & 1.000 \\
\hline
\end{tabular}

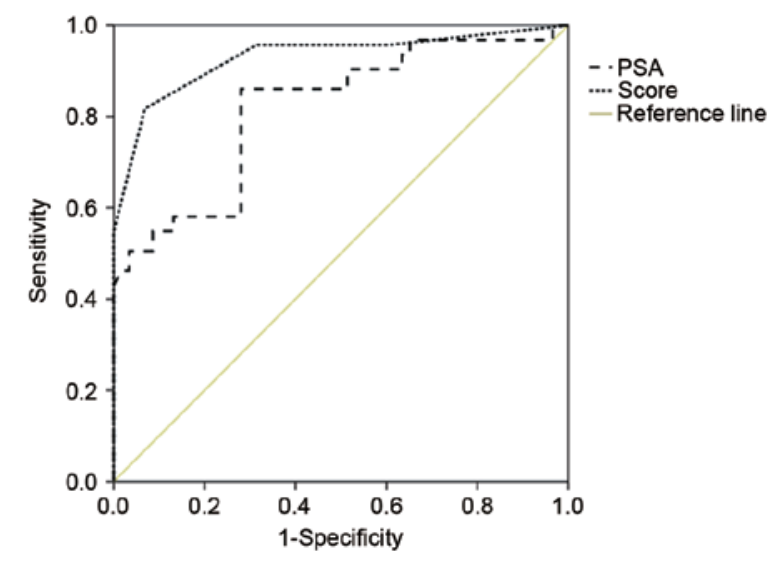

Figure 4. ROC curve of PSA and visually determined score. ROC identified a linear correlation between the visually determined score and PSA level $(\mathrm{r}=0.255, \mathrm{P}<0.001)$. PSA, prostate specific antigen; ROC, receiving operator characteristic.

According to the visual method of two scores, patients were divided into two groups as shown in Table VI. The table showed that when the cutoff for diagnosis used as the visually determined score was set to $\geq 2$ points, the positive predictive 
Table VI. Pathological diagnosis results when 2 points are set as the cutoff.

Pathological diagnosis

\begin{tabular}{lccc} 
Visually determined score & Non-prostate cancer & Prostate cancer & Total \\
\hline$<2$ (negative) & 120 & 4 & 124 \\
$\geq 2$ (positive) & 55 & 89 & 144 \\
Total & 175 & 93 & 268 \\
\hline
\end{tabular}

$\kappa=0.570, \mathrm{P}<0.01$ between the $<2$ scores group and $\geq 2$ scores group.

value of the diagnosis was $61.8 \%$ whereas the negative predictive value of the diagnosis was $96.8 \%$.

\section{Discussion}

Pathological examinations indicated that PCa specimens were solid and hard with a homogeneous texture and mostly gray-white or orange-yellow in color. Additionally, according to the PCa specimens in the current study, there were few hemorrhages and necrosis observed whereas normal prostate tissue is usually brown and porous (9,11-13,15-18).

A TRUS-guided biopsy is the primary method for clinically diagnosing PCa (8). The sextant biopsy scheme was initially proposed by Hodge et al (9) in 1989 and since then, this technique has been widely applied by clinicians However, studies have since determined that the detection rate of the sextant biopsy scheme is only $20-30 \%$, due to the smaller number of needle punctures performed $(19,20)$. Subsequently, a 12-core biopsy was created on the basis of the sextant biopsy scheme by adding one puncture point on each side of the bottom, middle and apex of the peripheral zone in the prostate of patients, and the positive detection rate subsequently increased to $44 \%$ (21). Furthermore, a 12-pointed puncture biopsy based prospective study conducted by Takenaka et al (22) demonstrated that the cancer detection rate was 47\%; significantly higher than that of the sextant biopsy scheme. Pepe and Aragona (23) performed a saturation prostate needle biopsy, utilizing 24-37 puncture needles, which had a detection rate twice that of the 12-pointed puncture. Therefore it seems that using more needles increases the detection rate (24).

PCa commonly occurs in older men (25), who often have other accompanying illnesses including hypertension, coronary disease and diabetes. In such patients, complications may occur following puncture biopsy. Mild complications can be resolved without treatment; however, serious ones may require surgical intervention (26). Common complications that occur during puncture are the vasovagal reflex, urethral hemorrhage, dysuria, acute urinary retention, hemospermia and infection (27). The vasovagal reflex manifests as palpitations, cold sweats and a drop in blood pressure, and a longer operation time increases the probability of developing this complication (28). A urethral hemorrhage (even heavy hemorrhage) occurs due to the damage of the cavernous body of the urethra and urinary mucosa, and requires surgical intervention (29). The incidence of severe complications, such as perineal rectofistula, is highly associated with the number of punctures performed. Increasing the number of punctures increases the positive diagnostic rate but also increases the risk of serious complications occurring (30-32).

In the present study, prostate specimens were visually inspected and scored, and the correlation between the visually determined scores and pathological results was determined. ROC identified a linear correlation between the visually determined score and PSA level. Furthermore, logistic regression analysis indicated that PAS and prostate biopsy specimen visual grading have statistical significance to distinguish between prostate cancer and non-prostate cancer patients. Furthermore, the accuracy of the visually determined score was higher than PSA in the diagnosis of PCa. When the visually determined score was set to 2 points, the sensitivity $(81.7 \%)$ and specificity $(93.1 \%)$ were relatively higher compared with when the score was set to 1 or 3 points. Among the 268 participants, the visually determined score of at least one specimen in 144 patients was $\geq 2$, of which 89 patients $(61.8 \%)$ were diagnosed with PCa. Therefore, the sextant biopsy scheme was used. For specimen scores that were $<2$, while PSA or imaging suggested PCa, a 12-pointed puncture method or saturation prostate needle biopsy was applied in order to obtain more samples to elevate the positive rate. For the scores of specimens $>2$ identified using the sextant biopsy scheme, it was deemed unnecessary to increase the number of puncture needles and thus the risk of complications and time of biopsy were reduced.

After applying this method in our hospital for the $\mathrm{PCa}$ biopsy, the positive rate was as high as $53.2 \%$, which is higher than $34.8 \%$ as previously reported (33). Furthermore, the incidence of postoperative hematuria was $21.3 \%$, which is lower than the $30 \%$ previous reported in the statistical analysis (34).

In conclusion, the current study demonstrated that the positive biopsy rate of PCa could be significantly improved. Additionally, under the sextant biopsy scheme rate of $\mathrm{PCa}$, the postoperative complications and repetition rate of puncture were significantly reduced. Future studies investigating the current method should include a greater number of participants.

\section{Acknowledgements}

The present study was funded by the Shen-kang Development Projects (grant no. SHDC12015912). 


\section{References}

1. Woodrum DA, Kawashima A, Gorny KR and Mynderse LA: Targeted prostate biopsy and MR-guided therapy for prostate cancer. Abdom Radiol (NY) 41: 877-888, 2016.

2. Siegel RL, Miller KD and Jemal A: Cancer statistics, 2015. CA Cancer J Clin 65: 5-29, 2015.

3. Woodrum DA, Kawashima A, Gorny KR and Mynderse LA: Prostate cancer: State of the art imaging and focal treatment. Clin Radiol: Apr 3, 2017 (Epub ahead of print).

4. Adeola HA, Blackburn JM, Rebbeck TR and Zerbini LF: Emerging proteomics biomarkers and prostate cancer burden in Africa. Oncotarget 8: 37991-38007, 2017.

5. Jemal A, Bray F, Center MM, Ferlay J, Ward E and Forman D: Global cancer statistics. CA Cancer J Clin 61: 69-90, 2011.

6. Djavan B, Zlotta A, Kratzik C, Remzi M, Seitz C, Schulman CC and Marberger M: PSA, PSA density, PSA density of transition zone, free/total PSA ratio, and PSA velocity for early detection of prostate cancer in men with serum PSA 2.5 to $4.0 \mathrm{ng} / \mathrm{ml}$. Urology 54: 517-522, 1999.

7. Pannek J and Partin AW: The role of PSA and percent free PSA for staging and prognosis prediction in clinically localized prostate cancer. Semin Urol Oncol 16: 100-105, 1998.

8. Paul R, Korzinek C, Necknig U, Niesel T, Alschibaja M, Leyh H and Hartung R: Influence of transrectal ultrasound probe on prostate cancer detection in transrectal ultrasound-guided sextant biopsy of prostate. Urology 64: 532-536, 2004.

9. Hodge KK, McNeal JE, Terris MK and Stamey TA. Random systematic versus directed ultrasound guided transrectal core biopsies of the prostate. J Urol 142: 71-74, 1989.

10. Presti JC: Prostate biopsy: Current status and limitations. Rev Urol 9: 93-98, 2007.

11. Scattoni V, Zlotta AR, Nava L, Roscigno M, Montorsi F and Rigatti P: Prostatic transrectal ultrasound (TRUS) guided biopsy schemes and TRUS prostatic lesion-guided biopsies. European Urol Suppl 1: 28-34, 2002.

12. Naughton CK, Miller DC, Mager DE, Ornstein DK and Catalona WJ: A prospective randomized trial comparing 6 versus 12 prostate biopsy cores: Impact on cancer detection. J Urol 164: 388-392, 2000

13. de la Taille A, Antiphon P, Salomon L, Cherfan M, Porcher R, Hoznek A, Saint F, Vordos D, Cicco A, Yiou R, et al: Prospective evaluation of a 21-sample needle biopsy procedure designed to improve the prostate cancer detection rate. Urology 61: 1181-1186, 2003.

14. Porcaro AB, Novella G, Mattevi D, Bizzotto L, Cacciamani G, Luyk ND, Tamanini I, Cerruto MA, Brunelli M and Artibani W: Chronic inflammation in prostate biopsy cores is an independent factor that lowers the risk of prostate cancer detection and is inversely associated with the number of positive cores in patients elected to a first biopsy. Curr Urol 9: 82-92, 2016.

15. Bostwick DG and Montironi R: Evaluating radical prostatectomy specimens: Therapeutic and prognostic importance. Virchows Arch 430: 1-16, 1997.

16. Hall GS, Kramer CE and Epstein JI: Evaluation of radical prostatectomy specimens: A comparative analysis of sampling methods. Am J Surg Pathol 16: 315-324, 1992.

17. McNeal JE, Price HM, Redwine EA, Freiha FS and Stamey TA: Stage A versus stage B adenocarcinoma of the prostate: Morphological comparison and biological significance. J Urol 139: 61-65, 1988.

18. Young RH; Armed Forces Institute of Pathology (US); Universities Associated for Research and Education in Pathology: Tumors of the Prostate Gland, Seminal Vesicles, Male Urethra and Penis. American Registry Pathology. Washington, DC, 2000.
19. Merrick GS, Gutman S, Andreini H, Taubenslag W, Lindert DL, Curtis R, Adamovich E, Anderson R, Allen Z, Butler W and Wallner K: Prostate cancer distribution in patients diagnosed by transperineal template-guided saturation biopsy. Eur Urol 52: 715-723, 2007.

20. Babaian RJ, Toi A, Kamoi K, Troncoso P, Sweet J, Evans R, Johnston D and Chen M: A comparative analysis of sextant and an extended 11-core multisite directed biopsy strategy. J Urol 163: $152-157,2000$.

21. Harvey CJ, Pilcher J, Richenberg J, Patel U and Frauscher F: Applications of transrectal ultrasound in prostate cancer. $\mathrm{Br} \mathrm{J}$ Radiol 85 (Spec No 1): S3-S17, 2012.

22. Takenaka A, Hara R, Ishimura T, Fujii T, Jo Y, Nagai A and Fujisawa M: A prospective randomized comparison of diagnostic efficacy between transperineal and transrectal 12-core prostate biopsy. Prostate Cancer Prostatic Dis 11: 134-138, 2008.

23. Pepe P and Aragona F: Saturation prostate needle biopsy and prostate cancer detection at initial and repeat evaluation. Urology 70: 1131-1135, 2007.

24. Yao MH, Zou LL, Wu R, Guo LH, Xu G, Xie J, Li P and Wang S: Transperineal ultrasound-guided 12-core prostate biopsy: An extended approach to diagnose transition zone prostate tumors. PLoS One 9: e89171, 2014.

25. Levinson A, Nagler EA and Lowe FC: Approach to management of clinically localized prostate cancer in patients with human immunodeficiency virus. Urology 65: 91-94, 2005.

26. Oranusi CK, Ugezu AI and Nwofor A: Diagnosis of prostate cancer with needle biopsy: Should all cases be biopsied before treatment? Niger J Clin Pract 15: 48-50, 2012.

27. Zhang XH, Jia Y, Guo WH, Ma YR, Yang J, Zhang Y, Hou SC and Zhang CC: Clinical comparison of the efficacy of three different bowel preparation methods on the infectious complications following transrectal ultrasonography guided prostate biopsy in nursing practice. J Clin Nurs: Apr 12, 2017 (Epub ahead of print).

28. Kam SC, Choi SM, Yoon S, Choi JH, Lee SH, Hwa JS, Chung KH and Hyun JS: Complications of transrectal ultrasound-guided prostate biopsy: Impact of prebiopsy enema. Korean J Urol 55: 732-736, 2014.

29. Krughoff K, Eid K, Phillips J, Stoimenova D, Smith D, O'Donnell C, Crawford ED and Barqawi A: The accuracy of prostate cancer localization diagnosed on transrectal ultrasound-guided biopsy compared to 3-dimensional transperineal approach. Adv Urol 2013: 249080, 2013.

30. Buskirk SJ, Pinkstaff DM, Petrou SP, Wehle MJ, Broderick GA, Young PR, Weigand SD, O'Brien PC and Igel TC: Acute urinary retention after transperineal template-guided prostate biopsy. Int J Radiat Oncol Biol Phys 59: 1360-1366, 2004.

31. Nomura T, Mimata H, Hata S, Yamasaki M, Tanaka E, Noguchi T, Mizutani A, Yoshitake S, Noguchi T and Nomura Y: Recto-peritoneal fistula following transperineal prostate biopsy. Int J Urol 12: 322-324, 2005.

32. Nafie S, Mellon JK, Dormer JP and Khan MA: The role of transperineal template prostate biopsies in prostate cancer diagnosis in biopsy naïve men with PSA less than $20 \mathrm{ng} \mathrm{ml}(-1$.). Prostate Cancer Prostatic Dis 17: 170-173, 2014.

33. Zhang Q, Wang W, Zhang B, Shi J, Fu Y, Li D, Guo S, Zhang S, Huang H, Jiang X, et al: Comparison of free-hand transperineal mpMRI/TRUS fusion-guided biopsy with transperineal 12-core systematic biopsy for the diagnosis of prostate cancer: A single-center prospective study in China. Int Urol Nephrol 49: 439-448, 2017.

34. Anastasiadis E, van der Meulen J and Emberton M: Hospital admissions after transrectal ultrasound-guided biopsy of the prostate in men diagnosed with prostate cancer: A database analysis in England. Int J Urol 22: 181-186, 2015. 